\title{
Interpretation of Contrast Dispersal Patterns by Experienced and Inexperienced Interventionalists
}

\author{
Matthew Smuck, MD, Zachary Abbott, DO, Eric Zemper, PhD
}

Objective: Determine how accurately inexperienced interventionalists interpret epidural contrast dispersal patterns compared with experienced interventionalists.

Design: Prospective comparative study.

Setting: University spine center.

Participants: Two experienced interventionalists and 3 pain fellows in training.

Methods: Each participant independently interpreted 100 images showing contrast dispersal patterns from transforaminal epidural injections. All images were obtained by another physician after $0.5 \mathrm{~mL}$ of contrast material was injected. The true contrast dispersal pattern was determined under live fluoroscopy during the injection and classified as follows: epidural only, vascular only, or simultaneous epidural and vascular. Participants were told to assume that $0.5 \mathrm{~mL}$ of contrast had been injected before each image and were asked to describe the contrast patterns as epidural, vascular, both, or neither.

Main Outcome Measure: Variance in agreement with the true contrast patterns between experienced and inexperienced participants.

Results: Overall, the inexperienced participants were in exact agreement with the true pattern in $52 \%$ of the images versus $70 \%$ for experienced participants $(P=.03)$. Experienced participants correctly identified epidural contrast patterns with greater accuracy than inexperienced participants (94\% and $76 \%$, respectively; $P=.01$ ). Not surprisingly, the accuracy for all participants was low in the identification of vascular patterns on these static images, with $73 \%$ accuracy for the experienced and $68 \%$ for the inexperienced $(P=.18)$.

Conclusion: Even with 6 months of intensive experience, trainees are significantly less accurate than experienced physicians in the interpretation of contrast dispersal patterns from transforaminal epidural injections. The competency of interventional trainees and the amount of experience necessary to safely perform epidural injections deserve further examination.

\section{INTRODUCTION}

The number of physicians performing spine injection procedures has been increasing; subsequently, the number of epidural injections performed annually has increased substantially. Between 1994 and 2001, the number of epidural injections performed by physiatrists increased by $1289 \%[1,2]$. The skills to perform these procedures are acquired through a variety of formal and informal training mediums. Nevertheless, there is a dearth of literature regarding the quantity and quality of training necessary to perform these procedures properly.

Transforaminal epidural injections of anesthetic and corticosteroids generally are safe, with a reported minor complication rate between $0.32 \%$ and $9.6 \%$, depending on the criteria used [3-5]. Rare but serious morbidity also has been documented, including subdural hematoma, transient paraplegia, brain or spinal cord infarction, and death [6-14]. Many of these adverse outcomes are thought to be secondary to inadvertent intravascular injection and embolization of corticosteroid particles via radiculomedullary arteries or the vertebral artery $[3,7-14]$. Therefore, the ability to recognize vascular contrast patterns is paramount to the safety of transforaminal epidural corticosteroid injections.

In addition, outcomes of epidural injections depend on the accurate delivery of therapeutic agents to the epidural space [15]. Consequently, interventionalists use contrast and
M.S. The Spine Program, Department of Physical Medicine and Rehabilitation, University of Michigan Health System, $325 \mathrm{E}$. Eisenhower Parkway, Suite 100, Ann Arbor, MI 48108. Address correspondence to: M.S.; e-mail: msmuck@med.umich.edu Disclosure: nothing to disclose

Z.A. The Spine Program, Department of Physical Medicine and Rehabilitation, University of Michigan Health System Disclosure: nothing to disclose

E.Z. The Spine Program, Department of Physical Medicine and Rehabilitation, University of Michigan Health System

Disclosure: nothing to disclose

Submitted for publication March 4, 2008; accepted June 4.

Disclosure Key can be found on the Table of Contents and at www.pmrjournal.org 
fluoroscopy to confirm proper contrast flow before injecting the treatment medication. Failure to correctly identify contrast patterns can result in nonepidural or intravascular injections, subsequently compromising the procedure's efficacy and safety.

Requirements for minimum experience with epidural injections during formal training programs are currently based on expert opinion. Still, there has been no attempt to objectively quantify the adequacy of these requirements. The purpose of this study was to investigate the amount of training necessary to accurately interpret contrast dispersal patterns. More specifically, it is designed to test the following hypothesis: physicians with 6 months' experience are less accurate than those with several years' experience in identifying contrast patterns from transforaminal epidural injections.

\section{METHODS}

Prospectively, the authors identified contrast dispersal patterns by observing the dynamic flow of contrast under live fluoroscopy during 50 epidural injections: 23 cervical and 27 lumbar. All injections were performed by a single practitioner (M.S.), a fellowship-trained, pain board-certified physician with 5 years' postfellowship experience. Digital subtraction angiography was not used during these injections. The study was approved by the University of Michigan Institutional Review Board and compliant with the Health Insurance Portability and Accountability Act (HIPAA).

Details of the cervical and lumbar epidural injection techniques were previously described $[16,17]$. During each injection, $0.5 \mathrm{~mL}$ of iohexol (Ominpaque 240, GE Healthcare Inc., Waukesha, WI) was injected under live fluoroscopy, and the dynamic flow was observed. Thus, the true contrast pattern for each injection was determined and recorded by the treating physician as follows: epidural only, vascular only, or simultaneous epidural and vascular. This recording determined the criterion standard contrast pattern for each injection. Additionally, 2 static images were saved from each injection. The first was the image automatically saved by the fluoroscope on release of the activation pedal simultaneous to the completion of the $0.5 \mathrm{~mL}$ contrast injection. The second image was a spot image taken 1 second after completing the contrast injection. With 2 images from each injection, a total of 100 images were obtained (Table 1).

All 100 images were assigned numbers, and then were randomly ordered by drawing the 100 numbers from a hat.

Table 1. Number of levels included in the 100 images viewed by experienced and inexperienced participants

\begin{tabular}{|c|c|c|c|}
\hline Cervical & $\mathrm{n}$ & Lumbar & $n$ \\
\hline C3/4 & 6 & $\mathrm{Ll} / 2$ & 2 \\
\hline $\mathrm{C} 4 / 5$ & 6 & L2/3 & 2 \\
\hline$C 5 / 6$ & 20 & L3/4 & 6 \\
\hline $\mathrm{Cb} / 7$ & 10 & $\llcorner 4 / 5$ & 16 \\
\hline $\mathrm{C} 7 / \mathrm{Tl}$ & 4 & L5/S1 & 28 \\
\hline
\end{tabular}

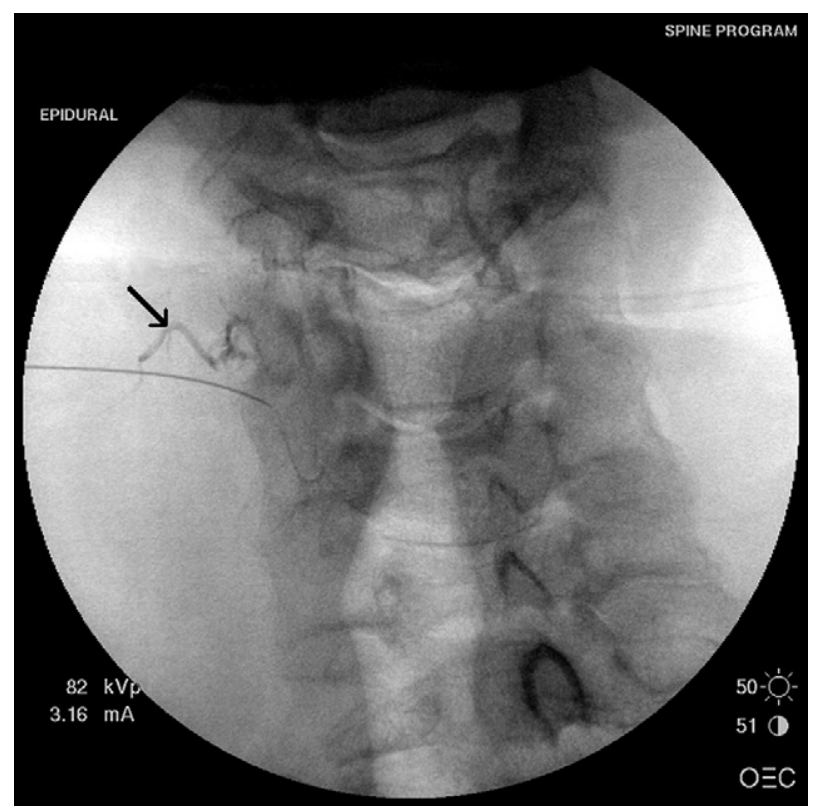

Figure 1. An example from the 100 fluoroscopy images where all 5 participants correctly identified the vascular contrast pattern. This image demonstrates vascular contrast spread (black arrow) during an attempted right C4-C5 transforaminal epidural injection. Interestingly, vascular injections were correctly identified by all 5 participants only in images in which the vascular injection occurred alone, that is, without simultaneous epidural contrast spread.

Five physician interventionalists voluntarily participated in the study. Each physician had experience using live and intermittent fluoroscopy and none of these 5 physicians was present during the actual performance of the injections included in this study. Two of these participants had more than 5 years of interventional spine experience (the experienced group); the remaining 3 were 6 months into an Accreditation Council for Graduate Medical Education (ACGME)-accredited pain fellowship (the inexperienced group) and had each participated in approximately 300 lumbar and 40 cervical fluoroscopically guided transforaminal injections.

These independent raters were instructed to progress through each of the 100 images without returning to previous images. They were also informed that $0.5 \mathrm{~mL}$ of contrast was injected into the patient before each image. Finally, they were asked to identify the contrast patterns on each image as epidural, vascular, both, or neither (Figure 1). Each of the 5 independent raters reviewed the images alone and recorded his or her findings on a corresponding numbered data collection form. The main outcome measure was variance in agreement with the true contrast patterns between the experienced and inexperienced participants. The data also were analyzed to identify potential causes of the variance.

To provide context regarding the clinical importance of errors by the participants, the errors were divided into 2 categories. Critical errors were defined as false-negative vascular (failure to identify a vascular contrast pattern when present in the true pattern) and false-positive epidural (iden- 


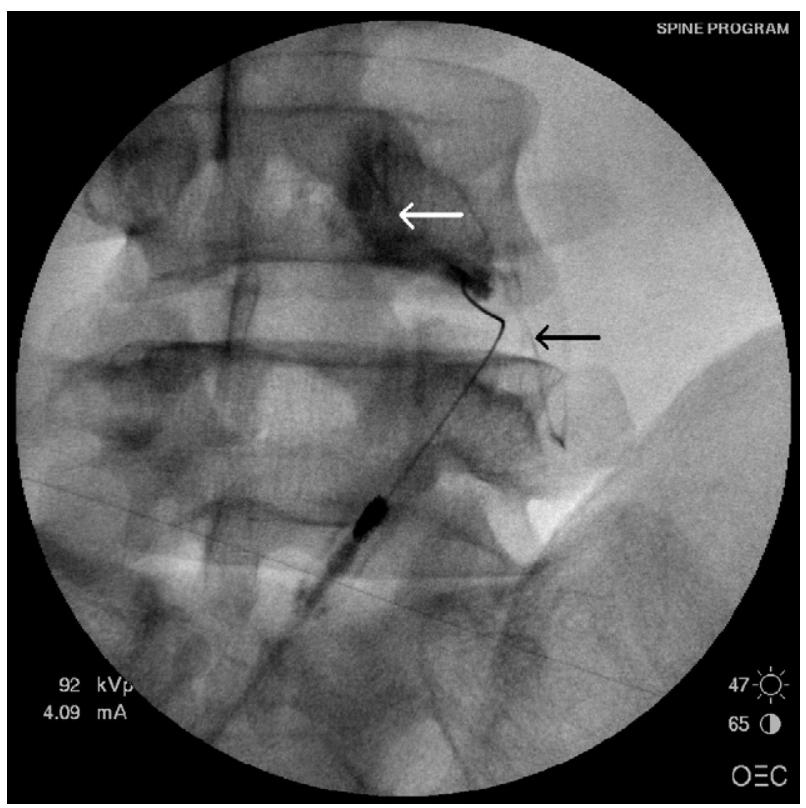

Figure 2. One example from the 100 fluoroscopy images presented to the 2 experienced and 3 inexperienced participants. This image demonstrates both epidural (white arrow) and vascular (black arrow) contrast flow during a right L4-L5 transforaminal epidural injection. For this particular image, all participants correctly identified the epidural contrast pattern. Although both of the experienced participants recognized the vascular pattern, only 1 of the 3 inexperienced participants identified it.

tification of an epidural pattern when not present in the true pattern). Noncritical errors were false-positive vascular and false-negative epidural.

All data were collected and analyzed by an independent third party. The mean agreement for each rater was computed versus the criterion standard, the true contrast pattern. Mean agreement with the criterion standard was expressed in percentages and calculated for multiple subgroups of images, including epidural only, vascular only, and simultaneous epidural and vascular. With the use of SAS software (Version 8 , Cary, NC), a paired sample t-test was used to test for statistical differences between mean values in the different subgroups.

\section{RESULTS}

Inexperienced participants were less accurate than experienced participants in their interpretations of the contrast patterns on the 100 images (Figure 2). Overall, exact agreement with the true pattern was $52 \%$ for the inexperienced versus $70 \%$ for the experienced participants $(P=.03)$. The greatest impact on overall accuracy for both groups was from errors identifying vascular contrast patterns. When examining only vascular contrast patterns, experienced participants did slightly better, $73 \%$ versus $68 \%$ accuracy, but the difference was not significant $(P=.18)$. With epidural contrast patterns, inexperienced participants were again less accurate $76 \%$ versus $94 \%(P=.01)$.

Interestingly, there was no difference in the number of critical errors between the 2 groups (24.3\% inexperienced versus $24.0 \%$ experienced, $P=.94$ ). Here again, the majority of these errors occurred in relation to vascular contrast patterns. When we compared experienced to inexperienced participants, we found that false-negative vascular errors were $23.0 \%$ and $23.7 \%$, respectively $(P=.90)$, and falsepositive epidural errors were $1.0 \%$ and $0.7 \%$, respectively $(P=.67)$.

The experienced group had significantly fewer noncritical errors than the inexperienced group, $8.5 \%$ versus $30.7 \%$ $(P=.01)$. False-negative epidural errors were $5.0 \%$ in the experienced group versus $22.7 \%$ in the inexperienced group $(P=.02)$, and false-positive vascular errors were $3.5 \%$ in the experienced group versus $8.0 \%$ in the inexperienced group $(P=.26)$. (See Table 2.)

\section{DISCUSSION}

According to the Medicare Part B claims database, the use of epidural injections has increased substantially during the last 15 years $[1,2]$. This increase is in part the result of an increasing number of physicians performing spine injection procedures. Still, there is a paucity of literature regarding the quantity or quality of training necessary to perform these injections competently. In this study, we examined the accuracy of physician interventionalists with 6 months of ACGME-accredited pain fellowship training in identifying contrast dispersal patterns during transforaminal epidural injections.

Under ideal conditions, one would expect the experienced participants to be in perfect agreement with the "true" contrast patterns. In this study, the experienced participants were not $100 \%$ accurate. They properly identified vascular contrast patterns $73 \%$ of the time and epidural patterns $94 \%$ of the time. Most likely, the primary cause of these deficits is the disadvantage created by interpreting static fluoroscopy

Table 2. Incidence of critical and noncritical errors comparing experienced and inexperienced participants and showing statistical significance of differences between means of the 2 groups

\begin{tabular}{lcrr}
\hline \multicolumn{1}{c}{ Type of Error } & Experienced, \% & Inexperienced, \% & P Value \\
\hline Critical Errors & & 23.7 & .7 \\
False-negative vascular & 23.0 & .90 \\
False-positive epidural & 1.0 & 8.0 & .67 \\
Noncritical Errors & 3.5 & 22.7 & .26 \\
False-positive vascular & 5.0 & .02 \\
False-negative epidural & & \\
\hline
\end{tabular}


images. Observing dynamic flow of contrast during live fluoroscopy provides visual feedback about epidural and vascular patterns that cannot be reproduced on static images. In fact, research has demonstrated that vascular contrast pattern recognition is reduced with intermittent static fluoroscopy [16]. Video clips of the contrast injections could have provided more details; unfortunately, the fluoroscope used in this study was not capable of saving video images.

Of course, a computer-based review of the final fluoroscopy images obtained from multiple epidural injections cannot reproduce the full context and experience of participating in the injections. The best study design would have all participants present during the injections to interpret the contrast flow simultaneously under live fluoroscopy. In addition, there would be a second treating physician present to determine the true contrast flow pattern instead of relying on a single treating physician as the gold standard. The size of the treatment room and the busy schedules of the participating physicians made such a study impractical.

Because we lacked the ability to save video fluoroscopy images, and with limited time and space for physicians in the treatment room, the only remaining option was to save static fluoroscopy images to present to the study participants at a later time. This intrinsic limitation in the study design likely reduced the accuracy of all of the study's participants. However, it was sufficient to test the primary hypothesis of this study. Because both the experienced and inexperienced participants were subject to the same limitations, the differences observed are relevant and attributable to their different levels of experience and not to other external factors.

Although inexperienced participants were less accurate in identifying epidural contrast patterns (76\% versus $94 \%$; $P=.01)$ and vascular contrast patterns (68\% versus $73 \%$; $P=.18$ ), the clinical relevance of these differences is not immediately clear. To draw more valuable conclusions, the results also were analyzed in terms of 2 distinct groups of errors: critical errors and noncritical errors (Table 2).

The participant's failure to recognize a vascular contrast pattern (false-negative vascular) was considered a critical error because it could lead to inadvertent intravascular injection of the treatment medications. When accidental intravascular injections occur, intra-arterial injections present the greatest danger and can lead to serious neurologic injury or death [3,7-14]. Fortunately, the majority of intravascular injections are venous and not arterial. Still, intravenous injections should be avoided because they cause medication to be carried away from the intended site. In this study, the difference in false-negative vascular errors between the experienced and inexperienced groups was statistically insignificant. Thus, interventionalists with only 6 months of intensive training were as able as experienced interventionalists to recognize intravascular injections when they occurred.

The participant's erroneous identification of an epidural pattern when not present (false-positive epidural) also was considered a critical error because it results in the injection of therapeutic agents into nontarget tissue. This reduces the diagnostic or therapeutic value of the intended treatment.
These errors of epidural addition were quite low and essentially the same for both the experienced and inexperienced participants. In this regard, inexperienced interventionalists were as accurate as experienced interventionalists.

Although the rates of critical errors were similar between the experienced and inexperienced groups, the latter made significantly more noncritical errors. Further study is required to determine why the inexperienced group had a significantly greater rate of missed epidural contrast patterns. One potential explanation is that it was because of their limited experience observing the variable forms of epidural contrast flow. Their tendency to overcall intravascular patterns may represent a hypervigilance inherent to their trainee status.

Although these noncritical errors do not have the same potential for morbidity as the critical errors, they are not without consequence. Either of the noncritical errors would cause the interventionalist to reposition the needle and again inject contrast. Once confirmed, the injection should be completed accurately. However, this action unnecessarily lengthens the procedure time, requires excess needle manipulation, and increases the amount of contrast material and fluoroscopy time used. Thus, the patient experiences additional discomfort, and both the interventionalist and the patient are exposed to increased radiation.

At the time of their participation in the present study, the inexperienced participants had each completed 6 months of training in an ACGME-accredited pain fellowship program. Each had participated in approximately 300 lumbar and 40 cervical fluoroscopically guided transforaminal injections. The current ACGME Pain Medicine fellowship guidelines suggest that trainees perform a minimum of 25 image-guided lumbar spine injections and 15 image-guided cervical spine injections. The results of the present study suggest that these minimum training guidelines are insufficient to prepare interventionalists to perform transforaminal epidural injections with maximal accuracy and efficiency.

\section{CONCLUSION}

Even with 6 months of intensive interventional experience with numbers that greatly exceed the ACGME Pain Medicine fellowship guidelines (nearly triple the recommended numbers for cervical and more than tenfold greater for lumbar injections), trainees are less accurate than experienced interventionalists in correctly interpreting contrast dispersal patterns from transforaminal epidural injections. Fortunately, significant differences are observed only in noncritical errors, resulting in only mildly increased risk. The competency of interventional spine trainees and the amount of experience necessary to safely perform epidural injections deserve further examination. Current ACGME Pain Medicine fellowship guidelines appear to be inadequate, significantly underestimating the number of injections required to maximize competency. In light of the potential consequences of the errors observed in this study, and until additional data become available, it is recommended that physicians performing 
these procedures have more than 6 months training under the supervision of experienced practitioners and that the minimum requirements in ACGME Pain Medicine fellowship guidelines be re-evaluated. Additionally, the authors of future studies should determine whether a significant difference remains between the inexperienced and experienced physicians at the end of a 12-month fellowship.

\section{ACKNOWLEDGMENTS}

The authors wish to thank Jennifer Miner and Sarah Lucey for their help with data analysis and manuscript preparation.

\section{REFERENCES}

1. Carrino JA, Morrison WB, Parker L, Schweitzer ME, Levin DC, Sunshine JH. Spinal injection procedures: volume, provider distribution, and reimbursement in the U.S. Medicare population from 1993 to 1999. Radiology 2002;225:723-729.

2. Friedly J, Chan L, Devo R. Increases in lumbosacral injections in the Medicare population: 1994 to 2001. Spine 2007;32:1754-1760.

3. Baker R, Dreyfuss P, Mercer S. Cervical transforaminal injection of corticosteroids into a radicular artery: a possible mechanism for spinal cord injury. Pain 2003;103:211-215.

4. Botwin K, Gruber RD, Bouchlas CG, Torres-Ramos FM, Freeman TL, Slaten WK. Complications of fluoroscopically guided transforaminal lumbar epidural injections. Arch Phys Med Rehabil 2000;81:10451050.

5. Derby R, Lee SH, Kim BJ, Chen Y, Seo KS. Complications following cervical epidural corticosteroid injections by expert interventionalists in 2003. Pain Phys 2004;7:445-449.
6. Brouwers PJ, Kottink EJ, Simon MA, Prevo RL. A cervical anterior spinal artery syndrome after diagnostic blockade of the right C6-nerve root. Pain 2001;91:397-399.

7. Houten JK, Errico TJ. Paraplegia after lumbosacral nerve root block: report of three cases. Spine J 2000;2:70-75.

8. Karasek M, Bogduk N. Temporary neurologic deficit after cervical transforaminal injection of local anesthetic. Pain Med 2004;5:202-205.

9. Ludwig MA, Burns SP. Spinal cord infarction following cervical transforaminal epidural injection: a case report. Spine 2005;30:E266-E268.

10. McLain RF, Fry M, Hecht ST. Transient paralysis associated with epidural corticosteroid injection. J Spinal Disord 1997;10:441-444.

11. McMillan MR, Crumpton C. Cortical blindness and neurologic injury complicating cervical transforaminal injection for cervical radiculopathy. Anesthesiology 2003;99:509-511.

12. Rozin L, Rozin $R$, Koehler $S A$, et al. Death during transforaminal epidural corticosteroid nerve root block (C7) due to perforation of the left vertebral artery. Am J Forensic Med Pathol 2003;24:351-355.

13. Somayaji HS, Saifuddin A, Casey AT, Briggs TW. Spinal cord infarction following therapeutic computer tomography-guided left L2 nerve root injection. Spine 2005;30:E106-E108.

14. Tiso RL, Cutler T, Catania JA, Whalen K. Adverse central nervous system sequelae after selective transforaminal block: the role of corticosteroids. Spine J 2004:4:468-474.

15. Thomas E, Cyteral C, Abiad L, Picot MC, Taourel P, Blotman F. Efficacy of transforaminal versus interspinous corticosteroid injection in discal radiculalgia - a prospective, randomised, double-blind study. Clin Rheumatol 2003;22:299-304.

16. Smuck M, Fuller B, Chiodo A, et al. Accuracy of intermittent fluoroscopy to detect intravascular injection during transforaminal epidural injections. Spine 2008;33:E205-E210.

17. Smuck M, Fuller B, Yoder B, Huerta J. Incidence of simultaneous epidural and vascular injection during lumbosacral transforaminal epidural injections. Spine J 2007;7:79-82. 